\title{
Annote: A Serious Game for Medical Students to Approach Lesion Skin Images of a Digital Library
}

\author{
Fabrizio Balducci \\ Dipartimento di Ingegneria "Enzo Ferrari" \\ Università degli Studi di Modena e Reggio Emilia \\ Via Vivarelli 10, Modena MO 41125, Italy \\ \{name. surname\}@unimore.it
}

\begin{abstract}
Nowadays it is claimed that one method to learn how to execute a task is to present it as a gaming activity: in this way a teacher can offer a safe and controlled environment for learners also arousing excitement and engagement. In this work we present the design of the serious game 'Annote', to exploit a medical digital library with the aim to help dermatologists to teach students how to approach the examination of skin lesion images to prevent melanomas.
\end{abstract}

Keywords: education, learning, serious game, skin images, gamification

\section{Introduction}

The Gamification process consists in the application of game-design elements and principles in non-game contexts [16]: it uses the game mechanics to improve skills and knowledge of a subject, also enhancing its engagement and excitement while performing a task that usually does not provides them. Referring to the Csíkszentmihályi $[12,19]$ and Chen [8] studies the sense of fun is strictly connected with the Flow theory characterized by the constant steady and balance between the challenge offered to gamers and the skills developed while facing them: in $[5,11,13]$ are studies about video semantic recognition while the evaluation of affective states and moods are in [3].

An Exergame identifies games that are also a form of exercise and involves the creation of a context in which the subject can use certain tools to replicate a series of real movements or tasks: they are used to counteract a sedentary activity, medical rehabilitation and promoting an active lifestyle and they are designed to provide immediate feedback to the player with the possibility of monitoring behaviors and biological parameters.

With the gamification technique it is possible to develop serious games which are games designed not only for the pure entertainment: this game genre is focused on the simulation feature with pedagogical purpose, by exploiting fun and competition while used in environments like defense, education [1], scientific exploration, health care [14], emergency management, city politics [17]. 


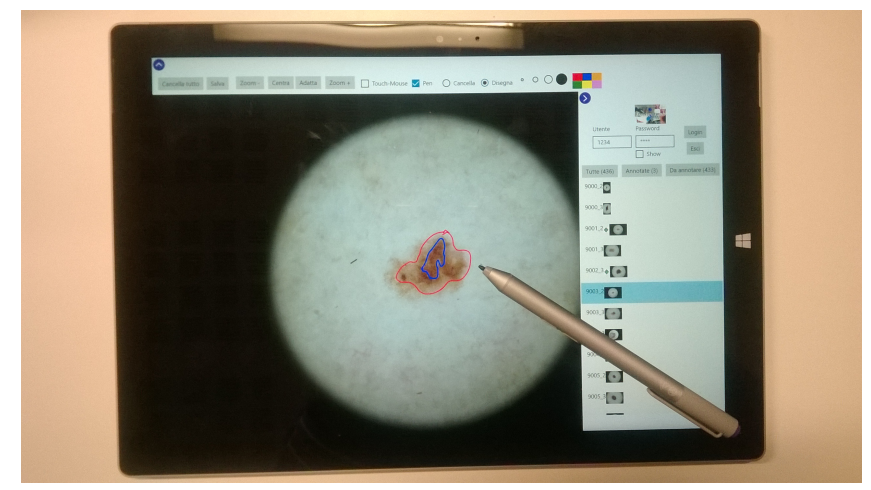

Fig. 1. The original annotation tool developed for Microsoft Surface Pro 3

The aim of this work is to apply the gamification process to one of the main activity in the daily work of dermatologists with which they make diagnoses and comparisons: the medical image annotation. The paper is organized as follows: Section 2 describes the general architecture and the design assumptions taken for the videogame while Section 3 shows technical details for the development of the prototype; finally conclusions and future work are illustrated in Section 4.

\section{The gamification process}

The idea to develop a serious (video)game for medical teaching (taking also inspiration from exergames) comes from the previous works for the development of an annotation tool [2] (Fig. 1) used by academic dermatologists to annotate skin lesion images and build an integrated medical Digital Library (Fig. 2).

The tool was designed following several interaction principles like affordance and direct manipulation [20,21]) with the aim to extract a dataset of visual features to use with Machine Learning techniques for a recommendation agent. During a preliminary test session, senior dermatologists and academic interns expressed interest towards innovative learning methods like serious gaming.

The survey of Hamari et al. [15] presents ten motivational affordances tested on empirical studies about gamification that will guide the design of Annote:

h1) Score points h2) Leaderboard h3) Achievements h4) Levels h5) Challenge h6) Story/Theme h7) Goals h8) Feedback h9) Rewards h10) Progress

The work of Coltell et al. [9] takes up the previous aspects and adds:

c1) Rules c2) Safety c3) Interaction c4) People c5) Fantasy c6) Exploration

The game objects is the act of 'draw strokes' and the right use of interactive tools: the repetitiveness is a learning element that, differently from commercial videogames, reinforces behavior change and progression in performances $[22,7]$.

The client-server architecture, the tools and the user interface (see Section 3) ensures that h8, c1, c2, c3 and c6 are met. A imaginative story (h6 and c5) 
results hard to introduce if not with the survival mission like "save lives as fast as possible" while there is a separate section with a non-interactive tutorial.

The main types of challenge offered by the game $(\mathrm{c} 1, \mathrm{~h} 5, \mathrm{~h} 7, \mathrm{~h} 10)$ are:

1. border challenge (precision): the player has to draw a lesion border annotation that imitates the 'official' one (ground-truth) also considering the completion of already begun strokes

2. structures challenge (recognition): the player has to annotate not lesion borders but groups of skin textures, clues and patterns (lines, circles, reticles,..)

3. time challenge (pressure): a variant of the previous two where the player must annotate respecting a flowing timer for each image

4. lesion classification (quiz): the player looks an image and gives a diagnosis on the severity of a lesion by choosing from a ranked set (Likert scale)

It must be noticed that the ground-truth has been previously made for the digital library by the academics (or by 'official' algorithms) so this guarantees the quality and the reliability of an annotation chosen for gaming comparison.

The variety of the game $(\mathrm{h} 4, \mathrm{~h} 5, \mathrm{~h} 7, \mathrm{~h} 10)$ is also given by the difficulty modes:

- the type and amount of images chosen by the teacher for a game session

- the activation of aid/impediment game features

To expand and diversify the gameplay, forms of rewards are introduced (h1, h2, h3, h5, h10, c4):

- power-up or penalty: they grant or steal resources to the player (time, points) or enable/disable features like the available tools (zoom, stroke width/color) or their performances (image resolution, mouse/pen speed)

- points and leaderboard: a centralized classification which emphasizes the desire to improve gaming (and learning) performances between students [18]

- personal profile: customizable, summarizes player informations

- badges and achievements: 'titles' that appear in the player profile and in the leaderboard screen; they depend by the number of accomplished tasks and by the amount of gained points

\section{Developing the serious game Annote}

This videogame allows to draw strokes on an image with different colors (8 choices) and pen sizes ( 4 choices); the dataset consists of 436 dermoscopic images in standard JPEG format with a resolution of 4000x2664 or 3000x4000 pixels. Large part of design and technology is in common with the original annotation tool and the use of the .NET Framework (ASP.NET with C\# the server side, XHTML with Javascript the client side) allows to neglect the problem of the input mode: since the client module reaches the server one by a standard web connection it is possible to interact with the Surface Pen if the client runs on a Surface device or with mouse/touch otherwise. 


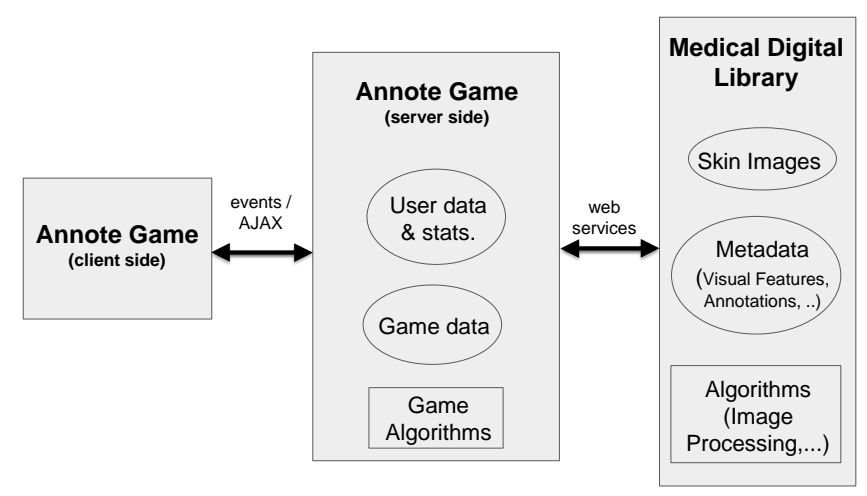

Fig. 2. The system architecture

In Fig. 2 there is the system architecture: a Digital Library manages the image collection, the ground-truth annotations and the algorithms for visual meta-data as in $[4,10,6]$; it interfaces with the game server using web services that allow communication between heterogeneous technologies; the game client permits the interaction with the user interface exploiting dynamic events and AJAX requests.

The Annote server manages data about the registered students with their points and achievements; the game data repository stores the settings of each game task while the game algorithms module performs evaluations (for example comparing an 'official ground-truth' annotations with that made by the player).

All gaming interactions and stroke collections are managed locally on the client and the data exchange with the server is limited to the original skin image, the drawn strokes and all the textual data (points, time, task requests, settings): this permits to minimize the network resources and to separate the management of data and algorithms from the module used by students to learn and to experiment on real medical data (that results protected for the privacy). In this prototype the user interface is minimal but functional and it is divided into three main sections (Fig. 3):

- upper section: shows the player profile (photo, nickname, points, badges, ...)

- middle section: tools to manage the image (Adapt, Center, Zoom) and to change the interaction mode (Erase or Draw) or the stroke features (color/width selection, annotation deleting)

- lower-left section: shows the main skin image with the superimposed strokes

- lower-right section: represents the interactive section of the game, in fact shows points and time (for time challenges), the setting of the task and the interactive messages occurred during the gaming session; moreover there are three buttons to commit, reload or change the game session.

At the beginning of a game session, an XML configuration file is send by the server to set up the user interface (for example to enable/disable buttons 


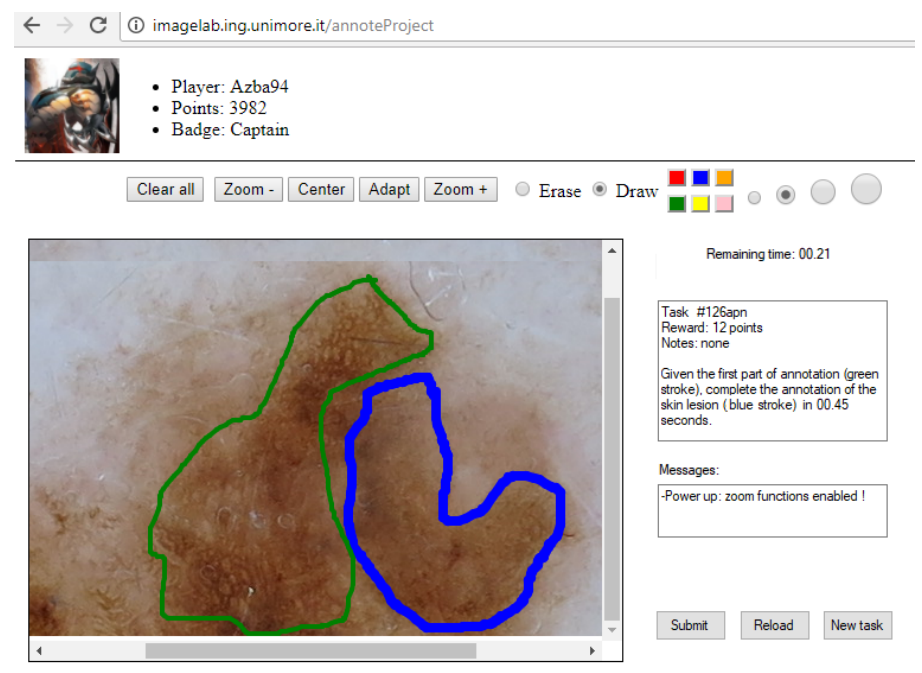

Fig. 3. The user interface of the Annote serious game

and widgets, instantiate the timer, load the image or draw default strokes). To manage game dynamics like power-up or the increasing difficulty, the game client implements a simple event manager that sends asynchronous messages to the server which in turn raises appropriate counter-events: for example, the end of the time involves a game failure, a zoom event will enable/disable the corresponding buttons, a time $X$ event will increase/decrease the timer by X seconds, a speed $X$ event will increase/decrease the mouse/pen speed by an amount of $\mathrm{X}$. When a student commits his work, the server algorithms will compute the corresponding rewards and will update the total points of the player in the leaderboard rank of the registered gamers.

\section{Conclusions and Future work}

In this paper, we exploited the interesting aspect of a pedagogical use of a digital library: help academic physicians in the delicate task of teaching, by transmitting a tacit knowledge hard to express with standard educational modes.

After the prototype presented here, it is necessary to design and perform evaluation studies on the field, involving directly large amount of medical students to obtain qualitative and quantitative data and compare the progressions between the 'standard' learning and the 'gamified' one. A usable editor can help to better customize the contents as well as services that address to academic resources when students have doubts; moreover sound and graphic effects with some forms of cooperative or multiplayer features can expand and improve the gaming experience. Finally, the expansion of this gamification process to other medical specializations that involve annotation protocols may well deserve further insights. 


\section{References}

1. Ardito, C., Buono, P., Costabile, M.F., Lanzilotti, R., Pederson, T.: Reexperiencing History in Archaeological Parks by Playing a Mobile Augmented Reality Game, pp. 357-366. Springer Berlin Heidelberg (2007)

2. Balducci, F., Borghi, G.: An Annotation Tool for a Digital Library System of Epidermal Data, pp. 173-186. Springer International Publishing (2017)

3. Balducci, F., Grana, C.: Affective Classification of Gaming Activities Coming from RPG Gaming Sessions, pp. 93-100. Springer International Publishing (2017)

4. Balducci, F., Grana, C.: Pixel Classification Methods to Detect Skin Lesions on Dermoscopic Medical Images, pp. 444-455. Springer (2017)

5. Baraldi, L., Grana, C., Cucchiara, R.: Recognizing and presenting the storytelling video structure with deep multimodal networks. IEEE TMM 19(5), 955-968 (2017)

6. Bolelli, F., Cancilla, M., Grana, C.: Two more strategies to speed up connected components labeling algorithms. In: International Conference on Image Analysis and Processing. pp. 48-58. Springer (2017)

7. Callaghan, M.J., McShane, N., Eguluz, A.G., Teills, T., Raspail, P.: Practical application of the learning mechanics-game mechanics (lm-gm) framework for serious games analysis in engineering education. In: 2016 13th International Conference on Remote Engineering and Virtual Instrumentation (REV). pp. 391-395 (Feb 2016)

8. Chen, J.: Flow in games (and everything else). Commun. ACM 50(4), 31-34 (2007)

9. Colteli, O., Grandi, X., Tosca, R., Latorre, P., Sánchez, J.S., Lizán, L.V., RosBernal, F., Martínez-Cadenas, C.: Designing serious games for learning support in medicine studies: A specific method to elicit and formalize requirements. In: Frontiers in Education Conference (FIE), 2014 IEEE. pp. 1-4. IEEE (2014)

10. Corbelli, A., Baraldi, L., Balducci, F., Grana, C., Cucchiara, R.: Layout Analysis and Content Classification in Digitized Books, pp. 153-165. Springer (2017)

11. Cornia, M., Baraldi, L., Serra, G., Cucchiara, R.: Visual Saliency for Image Captioning in New Multimedia Services. In: IEEE International Conference on Multimedia and Expo Workshops (2017)

12. Csikszentmihalyi, M.: Beyond boredom and anxiety. Jossey-Bass (2000)

13. Cucchiara, R., Grana, C., Prati, A.: Semantic transcoding for live video server. In: Proceedings of the 10th ACM Int. Conf. on Multimedia. MULTIMEDIA '02

14. Deponti, D., Maggiorini, D., Palazzi, C.E.: Smartphone's physiatric serious game. In: IEEE Int. Conf. on Serious Games and Applications for Health (2011)

15. Hamari, J., Koivisto, J., Sarsa, H.: Does gamification work?-a literature review of empirical studies on gamification. In: Hawaii Int. Conf. on System Sciences (2014)

16. Huotari, K., Hamari, J.: Defining gamification-a service marketing perspective. system 1(2), 3-4 (2012)

17. Maggiorini, D., Quadri, C., Ripamonti, L.A.: Opportunistic mobile games using public transportation systems: a deployability study. Multimedia Systems 20(5), 545-562 (2014)

18. Mäyrä, F.: An Introduction to Game Studies. SAGE Publications (2008)

19. Nakamura, J., Csikszentmihalyi, M.: The concept of flow. Handbook of positive psychology pp. 89-105 (2002)

20. Norman, D.A.: Affordance, conventions, and design. interactions 6(3), 38-43 (1999)

21. Shneiderman, B.: 1.1 direct manipulation: a step beyond programming languages. Sparks of innovation in human-computer interaction 17, 1993 (1993)

22. Ushaw, G., Eyre, J., Morgan, G.: A paradigm for the development of serious games for health as benefit delivery systems. In: Serious Games and Applications for Health (SeGAH), 2017 IEEE 5th International Conference on. pp. 1-8. IEEE (2017) 